Saudi Journal of Medicine

Abbreviated Key Title: Saudi J Med ISSN 2518-3389 (Print) |ISSN 2518-3397 (Online)

\title{
A Qualitative Analysis on Adult Psychology on Periodontal Health
}

\author{
Dr. Hima Varna, Bezawada ${ }^{1 *}$, Dr. Asma Shaik ${ }^{2}$, Dr. Swetha, Alugubelli ${ }^{3}$, Dr. Ravi Tejaswini Chunduri ${ }^{4}$, Dr. Asha Sri Sai \\ Sunkara ${ }^{5}$, Dr. Aliza Kapoor, BDS ${ }^{6}$ \\ ${ }^{1}$ MPH in epidemiology, Hudson College of Public Health, University of Oklahoma Health Sciences Center, Oklahoma, US \\ ${ }^{2}$ BDS, Sibar Institute of Dental Sciences, Guntur, AP, India \\ ${ }^{3}$ BDS, St. Joseph Dental College, Eluru, AP, India \\ ${ }^{4}$ BDS, Dayananda Sagar College of dental Sciences, Bengaluru, India \\ ${ }^{5}$ BDS, Dr. Sudha \& Nageswara Rao Siddhartha Institute of Dental Sciences, Andhra Pradesh, India \\ ${ }^{6}$ Rayat Bahra Dental College and Hospital, Punjab, India
}

DOI: $10.36348 / \mathrm{sjm} .2020 . \mathrm{v} 05 \mathrm{i} 03.007$

| Received: 03.03.2020 | Accepted: 15.03.2020 | Published: 21.03 .2020

*Corresponding author: Dr. Hima Varna, Bezawada

Abstract

\begin{abstract}
Aim: The purpose of the study is to analyze how periodontal health is affected based on psychological understanding of people about various periodontal diseases and if they are aware of basic hygiene modalities to keep a healthy periodontium. Materials and methods: A cross- sectional interview-based survey was conducted among 300 people between age groups of 20-60 years. During interviews, participants provided information on their demographic characteristics, educational attainment and general gingival health. They were also assessed on their level of stress that they usually endure. Result: Our study showed general increase periodontal as well as gingival diseases with age. People also had relatively less knowledge, mostly in those people who were not well educated. Young people were enthusiastic to improve their periodontal health. Conclusion: The study shows that people who are under greater stress showed a steep decline in their periodontal health and people with poor socio-economic background also ended up with more frequent oral health problems.
\end{abstract}

Keywords: Quality of life, Periodontitis, Psychology.

Copyright @ 2020: This is an open-access article distributed under the terms of the Creative Commons Attribution license which permits unrestricted use, distribution, and reproduction in any medium for non-commercial use (NonCommercial, or CC-BY-NC) provided the original author and source are credited.

\section{INTRODUCTION}

Oral health is stated as "a dentition that is contented, functional, and with such an advent that allows the people to accomplish their social functions and daily activities without physical, psychological or social inconveniences [1]." Oral disease has an impact on daily living and quality of life with physical, social and psychological impacts. Dentistry has mostly paid attention on assessment of treatment consequences rather than patient's experience of their disease [2].

Periodontal diseases including gingivitis and periodontitis are predominant in all populations worldwide. Severe periodontitis is estimated to affect $5 \%-20 \%$ of adults in both developed and developing countries. Moreover, gingivitis is universal in adults, and its signs are predominant as well among most children and adolescents [3].

The effect on quality of life of periodontal disease has recognized much less attention in comparison with other common oral conditions. An improved understanding of the effects of periodontal disease from individual's point of view is needed for the planning and assessment of public health interventions and for distribution of resources [4]. Chronic periodontal disease affects $65 \%$ of the Indian population and is considered to be cause of apprehension for oral health problems in country [5].

Recent evidence on the impact of periodontal disease on quality of life is limited to clinical studies [6], which limits the ability to oversimplify findings to extensive population. On the other hand, the few population studies were confined to precise age groups or controlled for a limited number of covariates [7]. Generally, people in rural areas disregard oral health as they lack awareness on dental diseases and also due to insufficient availability of dental services. Another reason which could be attributed is percentage of illiteracy common in the Indian population [8].

Quality of life (QoL) is concerned with "the degree to which a person appreciates the important possibilities of life [9]". Oral Health Impact Profile - 14 (OHIP14) is a well authenticated measure of OH QoL (Oral Heath Quality of Life) that detects dysfunction, 
distress and disability attributable to oral conditions based on WHO's "disease-impairment-disabilityhandicap" model. It may thus specify the effect of periodontal disease on a patient's welfare [10].

The World Health Organization (WHO) has upheld global oral health information bank using community periodontal index (CPI). This global oral health data from huge epidemiological studies from different countries which were collected to show the dispersal of periodontal disease in adolescents, adults and elderly populations. CPI index score varies from 0 to 4 and describes the periodontal ailment of individuals at population level. CPI score 0 represents no periodontal disease; score 1 means gingival bleeding on probing; score 2 shows the occurrence of calculus and bleeding; score 3 indicates shallow periodontal pockets of $4-5 \mathrm{~mm}$; score 4 signifies deep periodontal pockets of $6 \mathrm{~mm}$ or above[11].

\section{AIM}

This study aimed to assess knowledge of and attitudes towards periodontal health among adults and establish its relation to the basic psychological mindset of people as well their quality of life.

\section{MATERIALS AND METHODS}

The study used a cross-sectional, populationbased, interviewer-aided survey was done among subjects between ages 20 and 60 years in a population of 300 people and divided into two groups (20-39 years and 40-60 years). All patients who were physically and mentally capable of answering the questionnaire and gave informed consent for the study were included. Persons below the age of 20, suffering from systemic disease and having oral mucosal lesions, temporomandibular joint problems, Dental Fluorosis and Malocclusion were not included. During interviews, participants provided information on their demographic characteristics (sex and age), educational attainment and $\mathrm{OH}$ QoL.

$\mathrm{OH}$ QoL was measured using the oral health impact profile (OHIP-14), which contains 14 questions on the occurrence of adverse impacts caused by oral conditions. OHIP-14 items were categorized into seven variables: functional limitation (trouble speaking words and deteriorated taste), physical pain (discomfort eating foods), psychological discomfort (feeling self-conscious and feeling anxious), physical disability (intermittent meals and substandard diet), psychological disability (trouble in relaxing and awkwardness), social disability (irritability and trouble in doing usual jobs) and handicap (life less substantial and inability to function). Each person was asked to score each item on a 5-point ordinal scale coded 0 "'never', 1 "'hardly ever", 2 "occasionally", 3 "fairly often" and 4 "very often". The OHIP-14 score is the sum of answers and ranges from 0 to 56, with higher scores indicating poorer $\mathrm{OH}$ QoL[12] (Table 1).

Table-1: Oral Health Impact- 14 - it shows the 5-pointer score of the questionnaire as well groups of OHI

\begin{tabular}{|l|c|l|}
\hline \multicolumn{1}{|c|}{ OHI VARIABLES } & \multicolumn{2}{|c|}{ OHI SCORE } \\
\hline Functional Limitation & 0 & NEVER \\
\hline Physical Pain & 1 & HARDLY EVER \\
\hline Psychological Discomfort & 2 & OCCASIONALLY \\
\hline Psychological Disability & 4 & FAIRLY OFETN \\
\hline Social Disability & 5 & VERY OFTEN \\
\hline Handicap & \multicolumn{2}{|c|}{} \\
\hline
\end{tabular}

\section{STATISTICAL ANALYSIS}

The responses to the questions about periodontal knowledge and oral hygiene was scored for each participant. Cronbach's alpha was scored for each group based on oral health impact scores. All analysis was performed using a statistical software program SPSS (Statistical Package for the Social Sciences).

\section{RESULTS}

Cronbach's alpha for the OHIP-14 domains ranged from 0.54 to 0.62 and 0.34 to 0.66 for older age group (40-60 years) and younger age group (20-39 years) respectively (Table-2).

Table-2 Internal consistency reliability of OHIP-14 domains among young and old population

\begin{tabular}{|l|c|c|}
\hline OHI VARIABLES & \multicolumn{2}{|c|}{ CRONBACH'S ALPHA } \\
\hline & 20-39 years & 40-60 years \\
\hline Functional Limitation & 0.47 & 0.54 \\
\hline Physical Pain & 0.66 & 0.61 \\
\hline Psychological Discomfort & 0.62 & 0.57 \\
\hline Psychological Disability & 0.34 & 0.62 \\
\hline Social Disability & 0.54 & 0.56 \\
\hline Handicap & 0.48 & 0.61 \\
\hline
\end{tabular}


The study revealed that the prevalence of gingivitis and periodontitis was less in population with less age. It exhibited that loss of attachment gradually increases with age. Females showed increased bleeding tendency and periodontitis. The males exhibited a higher percentage of calculus and periodontal pocket formation.

The occurrence of periodontal pockets was observed to be higher in males. The higher prevalence of deep periodontal pockets among men may be due to their higher indulgence in adverse oral habits when compared to women. Age and gender were significantly associated with physical pain and physical disability areas. Mean psychological discomfort score decreased dramatically with age.

\section{DISCUSSION}

Now days, there has been growing interest in how periodontal disease and its treatment alters the well-being of patients [2]. Attitudes among our participants about the importance of oral and dental diseases in contrast with other systemic diseases, as well as the influence of gum disease on occupational and social relationships, were mainly positive. They also showed positive opinions toward preventive practices, including oral hygiene and regular check-ups. They usually thought that gum disease may occur even despite preventive care. This may demonstrate that they considered gum disease as not fully avoidable [3].

Chronic periodontitis disease is an immune- mediated inflammatory disease; the main etiology for disease is particular bacterial complexes in the oral biofilm. The disease may lead to attachment loss and alveolar bone in vulnerable patients. Pocket formation and/or gingival recession are major characteristic features clinically. Chronic periodontal disease affects $65 \%$ of the Indian population and is considered to be reason of concern for oral health complications in country [13].

Age- related rise in periodontal disease, observed in the current study follows the general inclination observed in the majority of the studies [14]. The strong association between age and periodontal destruction is mainly due to the effect of age as a replacement for the length of contact with etiologic factors [9].

It is also evident that psychological stress can hinder the cellular immune response. Communication between the central nervous system and the immune system and stress interrupts the homeostasis of this network, altering immune function.

Also, it is likely that systemic diseases related with periodontal disease such as diabetes, cardiovascular disease etc., may share psychosocial stress as common risk factor. Studies show the likely influence of psychosocial factors in the aetiology of inflammatory periodontal diseases [15]. Our results showed meagre periodontal knowledge. Health education programs should place more emphasis on causes and manifestations of periodontal disease [3].

\section{CONCLUSION}

As oral health frequently appears to be an issue of minor importance for Government and health policy makers, oral health care professionals should be at the forefront encouraging for resource deployment to improve access for proper oral health care. So, further research highlighting the need and method for incorporation of OHRQoL in treatment need, assessment system involving extensive sample representatives of general population, by aiming different age groups, stress levels and using different scales of OHRQoL should be directed. This will aid in planning services and for assessing outcomes in futher effectiveness trials of dental treatments from patient's perceptions.

\section{REFERENCES}

1. Gomes, A.S., Abegg, C., Fachel, J.M. (2009). Relationship between oral clinical conditions and daily performances. Braz Oral Res, 23:76-81.

2. Shah, M., Kumar, S.(2011). Improvement of Oral Health Related Quality of Life in Periodontitis Patients after Non-Surgical Periodontal Therapy. $J$. Int Oral Health, 3(6):16-22.

3. Gholami, M., Pakdaman, A., Jafari, A., Virtanen, J.I.(2014). Knowledge of and attitudes towards periodontal health among adults in Tehran. EMHJ, 20(3):196-202.

4. Allen, P. F.(2003). Assessment of oral health related quality of life. Health Qual Life Outcomes, 1:40.

5. Yoneyama, T., Okamoto, H., Lindhe, J., Socransky, S.S., $\quad$ Haffajee, A.D.(1988). Probing depth, attachment loss and gingival recession. $J$ ClinPeriodontol, 9:581- 91.

6. Reisine, S.T., Fertig, J., Weber, J., Leder, S.(1989). Impact of dental conditions on patients' quality of life. Community Dent Oral Epidemiol, 17(1):7-10.

7. Brennan, D.S., Spencer, A.J., Roberts-Thomson, K.F.(2007). Quality of life and disability weights associated with periodontal disease. J Dent Res, 86(8):713-17.

8. Ramoji, Rao, M.V., Katari, P.K., Vegi, L., Bypureddy, T.T., Prabhakara, Rao, K.S., Tejaswi, K.S.(2016). Prevalence of periodontal diseases among rural population of Mustabad, Krishna District. J Int Soc Prevent Communit Dent, 6:S5963.

9. Sanadhya, S., Aapaliya, P., Jain, S., Sharma, N., Choudhary, G., Dobaria, N.(2015). Assessment and comparison of clinical dental status and its impact on oral health-related quality of life among rural and urban adults of Udaipur, India: A crosssectional study. J Basic Clin Pharma, 6:50-58. 
10. Slade, G.D.(1997). Derivation and validation of a shortform oral health impact profile. Community Dent Oral Epidemiol, 25(4): 284-90.

11. World Health Organization. WHO Global Oral Health Data; 2005.

http://www.who.int/oral_health/databases/niigata/e n.

12. Slade, G.D.(1997). Derivation and validation of a shortform oral health impact profile. Community Dent Oral Epidemiol, 25(4): 284-90.

13. Yadav, T., Chopra, P., Kapoor, S.(2019). Association between chronic periodontitis and oral health-related quality of life in Indian adults. $J$ Int Oral Health, 11:280- 6.

14. Kumar, T.S., Dagli, R.J., Mathur, A., Jain, M., Balasubramanyam, G., Prabu, D.(2009). Oral health status and practices of dentate Bhil adult tribes of southern Rajasthan, India. Int Dent $J$, 59:133-40.

15. Goyal, S., Gupta, G., Thomas, B., Bhat, K.M., Bhat, G.S.(2013). Stress and periodontal disease: The link and logic!!. Ind Psychiatry J, 22(1):4-11. 